\title{
Blood stream infections-prevalence in a tertiary care institute, Central India
}

\author{
Prashant Meshram 1,*, Dilip Gedam², Nitin Ambhore², Rajesh Karyakarte ${ }^{4}$ \\ ${ }^{1}$ Tutor, ${ }^{2,3}$ Associate Professor, ${ }^{3}$ Professor, Dept. of Microbiology, Government Medical College, Akola, Maharashtra
}

*Corresponding Author:

Email: prshntmeshram187@gmail.com

\begin{abstract}
Introduction: Septicaemia is responsible for high morbidity and mortality in developing countries like India requiring prompt antimicrobial treatment for its proper management.

AIMS AND OBJECTIVES: Detection of etiological agent from blood culture of suspected septicaemia patients and it's antibiogram.

Material and Methods: A total of 227 blood culture samples were collected in Microbiology Laboratory during 1 July-2016 to 30 June-2017. Etiologic agents were isolated using conventional as well as automated blood culture methods and their antibiotic susceptibility was determined using standard protocol.

Results: Among clinically suspected septicemic patients 39(17.18\%) were culture positive. The most common organisms isolated were S. aureus(12), CONS(8), Klebsiella pneumoniae(5) and C. albicans(4) in decreasing order of their incidence. 75\% of S. aureus were methicillin resistant. All Gram positive organisms were sensitive to Glycopeptide antibiotics but showed variable resistance to other antibiotics. Gram negative bacteria were multidrug resistant with high degree of resistance towards Quinolones, Cephalosporins but a high sensitivity to Carbapenems. Only one non fermenter was resistant to Carbapenems which was sensitive to Colistin.

Conclusion: The use of automation can be a useful tool for isolation of rare organisms. The incidence of bacterial culture positivity is on the rise and fungal isolation is also common. In this era where over the counter drugs are easily available, drug abuse is difficult to control. But, having said that, formulation of Hospital Antibiotic Policy and its strict implementation is the need of the hour, which will eventually help us to control the menacing rise in antimicrobial resistance.
\end{abstract}

Keywords: Escherichia coli, Pseudomonas aeruginosa, Staphylococcus aureus.

\section{Introduction}

Septicemia is a life threatening emergency requiring rapid and aggressive antimicrobial treatment. ${ }^{1}$ Owing to rise in multidrug resistant strains of Klebsiella, Pseudomonas, Acinetobacter and Citrobacter species etc., they cause increased length of hospital stay and mortality in ICUs. ${ }^{1,2}$ The use of increased frequency in invasive procedures and increased use of invasive devices also contributes to this healthcare burden. ${ }^{2}$ Increase in blood stream infections can only be curbed by establishing baseline microbial resistance specific to the hospital preventing irrational use of antibiotics in that hospital and limiting antibiotic resistance. Therefore the study was undertaken to study bacteriological etiology of blood stream infections and antibiotic resistance pattern thereof. ${ }^{1}$

\section{Materials and Methods}

The study was conducted over a period of one year from 1 July-2016 to 30 June-2017 in the Department of Microbiology, GMC, Akola, a tertiary care 596 beded hospital. A total of 227 venous blood specimens were collected from suspected cases of septicaemia and
Pyrexia of Unknown Origin (PUO) patients admitted in ICUs, Wards following all the aseptic precautions and before administration of antibiotics. Relevant details of patients were filled in predesigned proforma. The study was conducted with approval from hospital ethics committee. Blood specimens were collected by procedure of venipuncture and inoculation into BacT Alert blood culture bottles (Biomerieux Ltd., France). The specimen was subcultured on BacT Alert positivity. The bottles were incubated for 7 days. The growth was identified by colony morphology, Gram stain of isolated colonies and conventional biochemical identification tests as per the standard protocol. ${ }^{1,2,3,4}$ the antibiotic resistance pattern of the isolated organisms were performed by Kirby Bauer Disc Diffusion Method on Muller Hinton agar plates and the results were recorded as per the Clinical and Laboratory Standards Institute 2016 Guidelines. ESBL and MBL producing strains were identified by Phenotypic Confirmatory Test and Double Disk Synergy Test respectively. The organisms difficult to identify using conventional blood culture methods were subjected to processing by Vitek 2 Compact ID/AST instrument (Biomerieux Ltd., France). 


\section{Observations}

Table1: Shows positivity of the blood culture

\begin{tabular}{|l|c|c|c|}
\hline \multicolumn{2}{|c|}{ Growth (\%) } & No Growth (\%) & Total (\%) \\
\hline Gram positive organisms & $23(58.97)$ & 188 & 227 \\
\cline { 1 - 2 } Gram negative organisms & $12(30.76)$ & & \\
\cline { 1 - 2 } Fungal spp. & $4(10.25)$ & & $227(100)$ \\
\hline Total & $39(17.18)$ & $188(82.82)$ & \\
\hline
\end{tabular}

Table 2: Shows distribution of the isolated organisms

\begin{tabular}{|c|c|c|c|}
\hline S. $\mathbf{N}$. & Organism & $\begin{array}{c}\text { Prevalence } \\
\text { (out of 39) }\end{array}$ & $\%$ \\
\hline \multicolumn{4}{|c|}{ Gram Positive Organisms } \\
\hline 1 & Staphylococcus aureus & 12 & 30.76 \\
\hline 2 & CONS & 8 & 20.51 \\
\hline 3 & Enterococcus spp & 3 & 7.69 \\
\hline \multicolumn{4}{|c|}{ Gram Negative Organisms } \\
\hline 4 & Klebsiella pneumoniae & 5 & 12.82 \\
\hline 5 & E. coli & 3 & 7.69 \\
\hline 6 & P. aeruginosa & 1 & 2.56 \\
\hline 7 & Acinetobacter spp. & 1 & 2.56 \\
\hline 8 & Stenotrophomonas maltophila & 1 & 2.56 \\
\hline 9 & Burkholderia cepacia & 1 & 2.56 \\
\hline \multicolumn{4}{|c|}{ Fungal SPP. } \\
\hline 10 & Candida albicans & 4 & 10.25 \\
\hline
\end{tabular}

227 blood samples were processed aerobically, among which 39(17.18\%) samples yielded growth. From 39 positive blood culture samples 35(89.74\%) yielded bacterial isolates and 4 (10.26\%) were Candida albicans. Only one sample yielded polymicrobial growth whereas all the others were monomicrobial. The only polymicrobial growth showed MRSA with Burkholderia cepacia. The unidentified species after conventional culture were subjected to identification by Vitek 2 Compact ID/AST instrument (Biomerieux Ltd., France). Among bacterial growth, Gram positive and gram negative isolates were 23(65.71\%) and $12(34.29 \%)$ respectively. MRSA was the predominant organism isolated followed by Coagulase Negative Staphylococci. In gram negative organisms Klebsiella pneumoniae (5) was the greatest. Other organisms found were candida albicans (4), MSSA (3), E. coli (3), Enterococcus faecalis (3), Pseudomonas aeruginosa(1), Acinetobacter spp.(1) Stenotrophomonas maltophila(1), Bukholderia cepacia (1) in decreasing order of prevalence respectively.

Inducible Clindamycin Resistance was seen in only one isolate of MRSA, whereas, all the gram positive isolates were found to be sensitive to Glycopeptide class of antibiotics. Coagulase Negative Staphylococcal spp. had relatively good sensitivity pattern than other gram positive organisms. 
Table 3: Antibiotic Sensitivity Pattern of Gram Positive Organisms

\begin{tabular}{|c|c|c|c|c|c|c|c|c|c|c|c|c|c|}
\hline Organism & P & Amp & Amc & Cd & Gm & Ak & Cip & E & Va & Lz & Co & Cu & Ctx \\
\hline MRSA(9) & 0 & 0 & 5 & 3 & 0 & 6 & 2 & 1 & 9 & 9 & 4 & - & - \\
\hline MSSA(3) & 0 & 1 & 2 & 1 & 1 & 2 & 1 & 0 & 3 & 3 & 2 & - & - \\
\hline CONS(8) & 0 & 5 & 9 & 9 & 8 & 9 & 6 & 6 & 8 & 8 & 9 & & \\
\hline $\begin{array}{c}\text { Enterococcus } \\
\text { spp.(3) }\end{array}$ & 1 & 1 & 1 & 2 & 1 & - & 2 & 1 & 3 & 3 & 3 & 1 & 3 \\
\hline
\end{tabular}

Table 4: Antibiotic Sensitivity Pattern of Gram Negative Organisms

\begin{tabular}{|c|c|c|c|c|c|c|c|c|c|c|c|c|c|c|c|c|}
\hline Organism & Amc & Gm & $\mathbf{A k}$ & Cip & $\mathbf{C u}$ & $\mathbf{C a}$ & Ctr & Ctx & Cpm & $\mathbf{C}$ & $\mathbf{P i}$ & $\mathbf{P t}$ & Cot & Im & $\mathbf{M p}$ & Col \\
\hline Klebsiella pneumoniae(5) & 2 & 3 & 4 & 2 & 1 & 3 & 3 & 3 & 3 & - & - & 3 & 3 & 4 & 4 & 5 \\
\hline E. $\operatorname{coli}(3)$ & 2 & 2 & 2 & 1 & 0 & 1 & 1 & 1 & 2 & - & - & 2 & - & 3 & - & \\
\hline P. aeruginosa $(1)$ & & 0 & 0 & 1 & - & - & - & 0 & 0 & & 0 & 0 & & 1 & & \\
\hline Acinetobacter spp.(1) & 0 & 0 & 1 & 0 & 0 & 1 & 1 & 1 & 1 & - & 0 & 0 & 1 & 1 & & \\
\hline $\begin{array}{l}\text { Stenotrophomonas } \\
\text { maltophila(1) }\end{array}$ & 0 & 0 & 0 & 0 & - & - & - & 0 & 0 & - & 0 & 0 & 1 & 0 & 0 & \\
\hline Burkholderia cepacia(1) & 0 & 1 & 1 & 1 & - & - & - & 1 & 1 & 1 & 1 & 1 & - & 1 & 1 & \\
\hline
\end{tabular}


Most of the isolates found in the study were multidrug resistant. Extended Spectrum $\beta$-Lcatamases were found in $K$. pneumoniae(1), E.coli(2), $P$. aeruginosa(1), whereas, Metallo $\beta$-Lactamases were seen in $K$. pneumoniae(1) and Stenotrophomonas spp.(1). The antibiotic susceptibility of rare organisms was confirmed by Vitek 2 Compact ID/AST instrument (Biomerieux Ltd., France).

\section{Discussion}

The blood stream infections are potentially difficult to treat and are costly as well. ${ }^{2}$ In our study, blood culture positivity was $17.18 \%$ which is comparable to study by Gulrez $\mathrm{M}$ et $\mathrm{al}^{13}(12.2 \%)$, Sharma et $\mathrm{al}^{14}$ (13.9\%), Roy et $\mathrm{al}^{15}(16.04 \%)$, Arora et $\mathrm{al}^{16}(20.02 \%)$ and Alam et $\mathrm{al}^{17}(20.9 \%)$. Whereas, Gohel $\mathrm{K}$ et al ${ }^{18}$ (9.2\%) showed lower culture positivity. A relatively low blood culture positivity in our study can be explained by patient receiving antibiotic from previous doctor or consumption of over the counter drug, similar with the findings of Gupta et al. ${ }^{1}$

In our study, the isolation rate of Gram positive organisms and Gram negative organisms was found to be $(58.97 \%)$, and $(30.76 \%)$, which was similar to Gohel $\mathrm{K}$ et $\mathrm{al}^{17}(58.3 \%)$ and $(40.2 \%)$ respectively. The remaining $(10.25 \%)$ were fungal species. The etiological profile may vary due to geographical locations, epidemiological variations and difference in etiologic agents, as previously described in Sahoo et al. ${ }^{12}$

Staphylococcal spp. were predminently isolated (51.28\%), which is similar to study of Magdy ${\mathrm{M} \mathrm{et} \mathrm{al}^{7}}^{7}$ but in contrast with the findings of Sahoo et $\mathrm{al}^{12}$ $(23.1 \%)$ and Sharma et $\mathrm{al}^{14}(28.5 \%)$. Also, CONS were $(20.51 \%)$ which was similar to study by Sharma et a ${ }^{14}$ (13.3\%). A low number of Enterococcal spp. were isolated in our study which was comparable to study of Mathur P et al. ${ }^{2}$

The isolation rate of Klebsiella pneumoniae (12.82\%) was highest among Gram Negative Organisms. E. coli and $P$. aeruginosa isolation rates were $(7.69 \%)$ and $(2.56 \%)$ which were similar to study of Sharma et al, ${ }^{14}$ i.e. (5.1\%) and (3.4\%) respectively. A single Acinetobacter spp. was also isolated which is consistant with findings of Sangwan et al ${ }^{20}$ Findings of Sahoo D et al ${ }^{12}$ were in contrast showed higher isolation rate of E.coli (34.6\%), Acinetobacter spp.(7.7\%) while no Pseudomonas spp. was isolated in his study.

4(10.25\%) Candida albicans were isolated from blood specimens which was comparable with findings of Pal et $\mathrm{al}^{8}$. Giri $\mathrm{S}$ et $\mathrm{al}^{19}$ showed use of long term antibiotics and intravascular catheter use directly correlated with a high incidence of Candida infection.

Gram Positive isolates showed more resistance towards Ampicillin and Erythromycin which was similar to studies of Gupta $\mathrm{S}$ et al, ${ }^{1}$ Devi $\mathrm{V}$ et al, ${ }^{5}$ Vanitha $\mathrm{RN}$ et al. ${ }^{9}$
Among twelve $S$. aureus isolates, nine were MRSA (75\%) which contradicted with the findings of Sharma et $\mathrm{al}^{14}$ which showed (32\%) Methicillin resistance. This might be due to unabated use of over the counter drugs and due to uncontrolled immigration of population from nearby villages to the city. High resistance was found against Enterococcal isolates which was similar to Jain $\mathrm{S}$ et al. ${ }^{10}$

Gram negative organisms were multidrug resistant in all the species. It might be due to lack of an appropriate antibiotic policy in the hospital. $33.3 \%$ of $E$. coli isolates were sensitive to Quinolones which was similar to previous studies as described by Gupta $S$ et al. ${ }^{1}$ A high degree of resistance to Aminoglycoside and $100 \%$ sensitivity to Carbapenems was shown by $P$. aeruginosa which was comparable to Radhakrishnan et al. ${ }^{11}$ It might be due to common use of Aminoglcoside and Cephalosporins as prophylaxis in the hospital.

We isolated rare species of bacteria using automated techniques, which were difficult to isolate using conventional methods. Again, the reports by automation were generated earlier than the conventional ones, highlighting its usefulness in treatment of the patients.

\section{Conclusion}

The chances of isolating pathogenic organism in a septicemic patient is higher in blood culture. But the use of automation can be a useful tool for isolation of rare organisms. The incidence of bacterial culture positivity is on the rise and fungal isolation is also common. Highly resistant Pseudomonas spp. can be a menace. Hence, examination and monitoring for resistant strains at definite time intervals should be done by Hospital Infection Control Committee. In this era where over the counter drugs are easily available, drug abuse is difficult to control. Therefore, formulation of Hospital Antibiotic Policy and its strict implementation is the need of the hour. It will certainly help the healthcare providers to control the rising antimicrobial resistance which is a serious problem these days.

\section{References}

1. Gupta S, Kashyap B. Bacteriological profile and antibiogram of blood culture isolates from a tertiary care hospital of North India. Trop J Med Res 2016;19:94

2. Mathur P, Varghese P,Tak V, Gunjiyal J, Lalwani S, Kumar S, Misra MC. Epidemiology of blood stream infections at a level-1 trauma care center of India. JLab Physicians2014; 6(1):22-7.

3. Collee JG, Fraser AG, Marmion BP, Simmons A. Tests for identification of Bacteria.In: Mackie and McCartney Practical Medical Microbiology. $14^{\text {th }}$ ed. London: Churchill Livingstone; 1996.p.131-49.

4. 4. Clinical and Laboratory Standards Institute. Performance Standards for Antimicrobial Susceptibility Testing. Twenty-Second Informational Supplement. Wayne, PA, USA: Clinical and Laboratory Standards Institute; 2012. p. M100-S22. 
5. Devi V, Sahoo B, Damrolien S, Praveen S, Lungran P, Devi M. A study on the bacterial profile of bloodstream infections in Rims Hospital. J Dent Med Sci 2015;14:18-23.

6. Mehta M, Dutta P, Gupta V. Antimicrobial susceptibility pattern of blood isolates from a teaching hospital in north India. Jpn J Infect Dis 2005;58:1746.

7. Magdy MM, Bialy AA, Ahmd AA. Methicillinresistant Staphylococcus aureus: A challenge for infection control. Menoufia Med J 2017;29:812-7.

8. Pal N, Sharma R, Rishi S, Vyas L. Optimum time to detection of bacteria and yeast species with BACTEC 9120 culture system from blood and sterile body fluids. J Lab Physic 2009;1(2):69-72.

9. 9. Vanitha RN, Kannan G, Venkata NM, Vishwakanth D, Nagesh VR, Yogitha M, et al. A retrospective study on blood stream infections and antibiotic susceptibility patterns in a tertiary care teaching hospital. Int J Pharm Pharm Sci 2012;4:543-8.

10. 10. Jain S, Kumar A, Kashyap B, Kaur IR. Clinico-epidemiological profil and high-level aminoglycoside resistance in enterococcal septicemia from a tertiary care hospital in east Delhi. Int J Appl Basic Med Res 2011;1:80-3.

11. 11. Radhakrishnan, V., et al. "Bloodstream infections in pediatric patients at cancer institute, Chennai." Indian journal of cancer 51.4 (2014): 418

12. 12. Sahoo D, Mohanty L, Panda S S, Mishra S N. Bacteriological analysis of blood culture isolates in patients with sepsis in a tertiary care hospital of eastern India. International Journal of Contemporary Medical Research. 2016;3:3448-50.

13. 13. Gulrez $\mathrm{M}$ et al. Spectrum of microorganisms isolated from blood culture and their resistance pattern. ERA's Journal of medical research. 2(1).(abstract)

14. 14.Sharma M, Goel N, Chaudhary U, Aggarwal R, Arora DR. Bacteraemia in children. Indian J Pediatr 2002;69:1029-32.

15. 15. Roy I, Jain A, Kumar M, Agarwal SK. Bacteriology of neonatal septicaemia in a tertiary care hospital of Northern India. Indian JMed Microbiol. 2002;20:156-9.

16. 16. Arora U, Devi P. Bacterial profile of blood stream infections and antibiotic resistance pattern of isolates. JK Science. 2007;9:186-90.

17. 17. Alam MS, Pillai PK, Kapur P, Pillai KK. Resistant patterns of bacteria isolated from bloodstream infections at a university hospital in Delhi. J Pharm Bioallied Sci. 2011;3:525-30.

18. 18. Gohel K, Jojera A, Soni S, Gang S, Sabnis R, Desai M. Bacteriological Profile and Drug Resistance Patterns of Blood Culture Isolates in a Tertiary Care Nephrourology Teaching Institute. BioMed Research International. 2014:1-5.

19. 19. Giri S, Kindo AJ: A review of Candida species causing blood stream infection. Indian J Med Microbiol. 2012, 30 (3):270-8.

20. 20. Sangwan J, Mane P, Vohra P, Ladhwal S, Malik AK. Bacteriologic Profile and Antmicrobial Resistance of Blood Culture Isolates of Septicemic Patients from Various Intensive Care Units in a Teaching Tertiary Care Institute of Haryana, India. Int. J. Curr. Microbiol. App. Sci. 2016;5(7):599-608.
How to cite this article: Meshram $\mathrm{P}$, Gedam D, Ambhore N, Karyakarte R. Blood stream infectionsprevalence in a tertiary care institute, Central India. Indian J Microbiol Res 2018;5(1):87-91. 